\title{
Strong coupling Hartree-Fock approximation in the dynamical mean-field theory
}

\author{
A.M.Shvaika \\ Institute for Condensed Matter Physics \\ of the National Academy of Sciences of Ukraine, \\ 1 Svientsitskii Str., 79011 Lviv, Ukraine
}

Received August 23, 2000

In the limit of infinite spatial dimensions, a thermodynamically consistent theory, which is valid for arbitrary value of the Coulombic interaction $(U<$ $\infty$ ), is built for the Hubbard model when the total auxiliary single-site problem exactly splits into four subspaces with different "vacuum states". Some analytical results are given for the Hartree-Fock approximation when the 4pole structure for Green's function is obtained: two poles describe contribution from the Fermi liquid component, which is ferromagnetic and dominant for small electron and hole concentrations ("overdoped case" of high- $T_{\mathrm{c}}$ 's), whereas other two describe contribution from the non-Fermi liquid, which is antiferromagnetic and dominant close to half filling ("underdoped case").

Key words: dynamical mean-field theory, Hartree-Fock approximation, Hubbard model, (anti)ferromagnetism, strong coupling

PACS: $71.10 . F d, 71.15 . \mathrm{Mb}, 05.30 . F k, 71.27 .+a$

\section{Introduction}

In the last decade, essential achievements in the theory of strongly correlated electron systems are connected with the development of the dynamical mean-field theory (DMFT) proposed by Metzner and Vollhardt [1] for the Hubbard model (see also $[2,3]$ and references therein). There are no restrictions on the $U$ value within this theory and it turns out to be useful for intermediate coupling $(U \sim t)$ for which it ensures a correct description of the metal-insulator phase transition and determines the region of the Fermi-liquid behaviour of the electron subsystem. Moreover, some classes of binary-alloy-type models (e.g., the Falicov-Kimball model) can be studied almost analytically within DMFT [4]. But in the case of the Hubbard model, the treatment of the effective single impurity Anderson model is very complicated and mainly computer simulations are used, which calls for the development of analytical approaches [5]. 
Such approaches can be built with a systematic perturbation expansion in terms of the electron hopping [6] using a diagrammatic technique for Hubbard operators $[7,8]$. One of them was proposed for the Hubbard $(U=\infty$ limit) and $t-J$ models [9]. The lack of such an approach is connected with the concept of a "hierarchy" system for the Hubbard operators when the form of the diagrammatic series and the final results strongly depend on the system of the pairing priority for Hubbard operators. On the other hand, it is difficult to generalize it to the case of arbitrary $U$.

In our previous paper [10] we developed, for the Hubbard-type models, a rigorous perturbation theory scheme in terms of electron hopping which is based on the Wick theorem for Hubbard operators [7,8], which is valid for arbitrary values of $U$ $(U<\infty)$ and which does not depend on the "hierarchy" system for $X$ operators. In the limit of infinite spatial dimensions, this analytical scheme permits us to build a self-consistent Kadanoff-Baym type theory [11] for the Hubbard model and some analytical results are given for simple approximations. Here we shall consider possible magnetic orderings in the Hartree-Fock type approximation.

\section{Perturbation theory in terms of electron hopping}

We consider the lattice electronic system described by the statistical operator:

$$
\hat{\rho}=\mathrm{e}^{-\beta \hat{H}_{0}} \hat{\sigma}(\beta), \quad \hat{\sigma}(\beta)=T \exp \left\{-\int_{0}^{\beta} \mathrm{d} \tau \int_{0}^{\beta} \mathrm{d} \tau^{\prime} \sum_{i j \sigma} t_{i j}^{\sigma}\left(\tau-\tau^{\prime}\right) a_{i \sigma}^{\dagger}(\tau) a_{j \sigma}\left(\tau^{\prime}\right)\right\},
$$

where $\hat{H}_{0}=\sum_{i} \hat{H}_{i}$ is a sum of the single-site contributions, and for the Hubbard model we must put

$$
H_{i}=U n_{i \uparrow} n_{i \downarrow}-\mu\left(n_{i \uparrow}+n_{i \downarrow}\right)-h\left(n_{i \uparrow}-n_{i \downarrow}\right), \quad t_{i j}^{\sigma}\left(\tau-\tau^{\prime}\right)=t_{i j} \delta\left(\tau-\tau^{\prime}\right) .
$$

It is supposed that we know the eigenvalues and eigenstates of the zero-order Hamiltonian: $H_{i}|i, p\rangle=\lambda_{p}|i, p\rangle$, and one can introduce Hubbard operators $\hat{X}_{i}^{p q}=$ $|i, p\rangle\langle i, q|$ in terms of which the zero-order Hamiltonian is diagonal

$$
H_{0}=\sum_{i} \sum_{p} \lambda_{p} \hat{X}_{i}^{p p}
$$

For the Hubbard model we have four states $|i, p\rangle=\left|i, n_{i \uparrow}, n_{i \downarrow}\right\rangle:|i, 0\rangle=|i, 0,0\rangle$ (empty site), $|i, 2\rangle=|i, 1,1\rangle$ (double occupied site), $|i, \uparrow\rangle=|i, 1,0\rangle$ and $|i, \downarrow\rangle=$ $|i, 0,1\rangle$ (sites with spin-up and spin-down electrons) with energies $\lambda_{0}=0, \lambda_{2}=$ $U-2 \mu, \lambda_{\downarrow}=h-\mu$, and $\lambda_{\uparrow}=-h-\mu$. The connection between the electron operators and the Hubbard operators is the following:

$$
n_{i \sigma}=X_{i}^{22}+X_{i}^{\sigma \sigma} ; \quad a_{i \sigma}=X_{i}^{0 \sigma}+\sigma X_{i}^{\bar{\sigma} 2} .
$$

The expression for $\langle\sigma(\beta)\rangle_{0}$ is a series of terms that are products of the hopping integrals and averages of the electron creation and annihilation operators or Hubbard 
operators, that are calculated with the use of the corresponding Wick's theorem $[7,8]$, and can be written as [10]:

$$
\langle\hat{\sigma}(\beta)\rangle_{0}=\left\langle\exp \left\{-\frac{1}{2}\right\}\right.
$$

where arrows denote the zero-order Green's functions

$$
g_{p q}\left(\omega_{n}\right)=\frac{1}{\mathrm{i} \omega_{n}-\lambda_{p q}},
$$

wavy lines denote hopping integrals and $\square, \ldots$ stand for complicated " $n$ vertices". Each vertex (many-particle single-site Green's function) is multiplied by a diagonal Hubbard operator denoted by a circle.

\section{Irreducible many-particle Green's functions}

For the Hubbard model, expressions for the two-vertex

$$
\bigoplus=\sum_{p} \hat{X}_{i}^{p p} g_{\sigma(p)}\left(\omega_{n}\right)
$$

the four-vertex

$$
\overline{0}=\sum_{p} \hat{X}_{i}^{p p} g_{\sigma(p)}\left(\omega_{n}\right) g_{\sigma(p)}\left(\omega_{n+m}\right) \widetilde{U}_{\sigma \bar{\sigma}(p)}\left(\omega_{n}, \omega_{l} \mid \omega_{m}\right) g_{\bar{\sigma}(p)}\left(\omega_{l}\right) g_{\bar{\sigma}(p)}\left(\omega_{l+m}\right)
$$

and for the vertices of higher order possess one significant feature [10]. They decompose into four terms with different diagonal Hubbard operators $X^{p p}$, which project our single-site problem onto certain "vacuum" states (subspaces), and zero-order Green's functions

$$
g_{\sigma(p)}\left(\omega_{n}\right)=\left\{\begin{array}{ll}
g_{\sigma 0}\left(\omega_{n}\right) & \text { for } p=0, \sigma \\
g_{2 \bar{\sigma}}\left(\omega_{n}\right) & \text { for } p=\bar{\sigma}, 2
\end{array},\right.
$$

which describe all possible excitations and scattering processes around these "vacuum" states. Here

$$
\widetilde{U}_{\sigma \bar{\sigma}(p)}\left(\omega_{n}, \omega_{l} \mid \omega_{m}\right)= \begin{cases}U \pm U^{2} g_{20}\left(\omega_{n+l+m}\right) & \text { for } p=0,2 \\ U \pm U^{2} g_{\sigma \bar{\sigma}}\left(\omega_{n-l}\right) & \text { for } p=\sigma, \bar{\sigma}\end{cases}
$$

is a renormalized Coulombic interaction in the subspaces. In diagrammatic notations, expression (3.2) can be represented as

$$
\square=Y x \pm
$$


where dots denote the Coulombic correlation energy $U$ and the dashed arrows denote bosonic zero-order Green's functions: doublon $g_{20}\left(\omega_{m}\right)$ or magnon $g_{\sigma \bar{\sigma}}\left(\omega_{m}\right)$. The contributions to the six-vertex can be presented by the following diagrams:

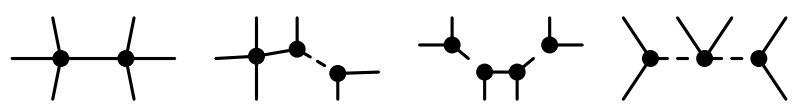

where the first three diagrams contain the internal vertices of the same type as in equation (3.5). So, we can introduce primitive vertices $\rangle, \mathbf{\gamma}, \mathbf{x}$, by which one can construct all $n$ vertices in the expansion $(2.5){ }^{1}$

\section{Dynamical mean-field theory}

Within the framework of the considered perturbation theory in terms of electron hopping a single-electron Green's function can be presented in the form

$$
G_{\sigma}\left(\omega_{n}, \boldsymbol{k}\right)=\frac{1}{\Xi_{\sigma}^{-1}\left(\omega_{n}, \boldsymbol{k}\right)-t_{\boldsymbol{k}}}
$$

where we introduce an irreducible part $\Xi_{\sigma}\left(\omega_{n}, \boldsymbol{k}\right)$ of Green's function which, in general, is not local. In the case of infinite dimensions $d \rightarrow \infty$ one should scale the hopping integral according to $t_{i j} \rightarrow t_{i j} / \sqrt{d}$ in order to obtain finite density-of-states and it was shown by Metzner in his pioneer work [12] that in this limit the irreducible part becomes local $\Xi_{i j \sigma}\left(\tau-\tau^{\prime}\right)=\delta_{i j} \Xi_{\sigma}\left(\tau-\tau^{\prime}\right)$ or $\Xi_{\sigma}\left(\omega_{n}, \boldsymbol{k}\right)=\Xi_{\sigma}\left(\omega_{n}\right)$ and such a site-diagonal function can be calculated by mapping the $d \rightarrow \infty$ lattice problem (2.1) onto the atomic model with the auxiliary Kadanoff-Baym field [4]

$$
t_{i j}^{\sigma}\left(\tau-\tau^{\prime}\right)=\delta_{i j} J_{\sigma}\left(\tau-\tau^{\prime}\right)
$$

The self-consistent set of equations for $\Xi_{\sigma}\left(\omega_{n}\right)$ and $J_{\sigma}\left(\omega_{n}\right)$ (e.g., see [3] and references therein) is the following:

$$
\frac{1}{N} \sum_{\boldsymbol{k}} \frac{1}{\Xi_{\sigma}^{-1}\left(\omega_{n}\right)-t_{\boldsymbol{k}}}=\frac{1}{\Xi_{\sigma}^{-1}\left(\omega_{n}\right)-J_{\sigma}\left(\omega_{n}\right)}=G_{\sigma}^{(a)}\left(\omega_{n},\left\{J_{\sigma}\left(\omega_{n}\right)\right\}\right),
$$

where $G_{\sigma}^{(a)}\left(\omega_{n},\left\{J_{\sigma}\left(\omega_{n}\right)\right\}\right)$ is the Green's function for the atomic limit (4.2).

The grand canonical potential for the lattice is connected with the one for the atomic limit by the expression [4]

$$
\frac{\Omega}{N}=\Omega_{a}-\frac{1}{\beta} \sum_{n \sigma}\left\{\ln G_{\sigma}^{(a)}\left(\omega_{n}\right)-\frac{1}{N} \sum_{\boldsymbol{k}} \ln G_{\sigma}\left(\omega_{n}, \boldsymbol{k}\right)\right\} .
$$

On the other hand, we can write, for the grand canonical potential for the atomic limit $\Omega_{a}$, the same expansion as in equation (2.5) but with diagonal $X$ operators at

\footnotetext{
${ }^{1}$ For $n$ vertices of higher order a new primitive vertices can appear but we do not check this due to the rapid increase of the algebraic calculations with the increase of $n$.
} 
the same site. We can reduce their product to a single $X$ operator that can be taken outside the exponent in (2.5), and its average is equal to $\left\langle X^{p p}\right\rangle_{0}=\mathrm{e}^{-\beta \lambda_{p}} / \sum_{q} \mathrm{e}^{-\beta \lambda_{q}}$. Finally, for the grand canonical potential for the atomic limit we get [10]

$$
\Omega_{a}=-\frac{1}{\beta} \ln \sum_{p} \mathrm{e}^{-\beta \Omega_{(p)}}
$$

where $\Omega_{(p)}$ are the "grand canonical potentials" for the subspaces.

Now we can find the single-electron Green's function for the atomic limit by

$$
G_{\sigma}^{(a)}\left(\tau-\tau^{\prime}\right)=\frac{\delta \Omega_{a}}{\delta J_{\sigma}\left(\tau-\tau^{\prime}\right)}=\sum_{p} w_{p} G_{\sigma(p)}\left(\tau-\tau^{\prime}\right)
$$

where $G_{\sigma(p)}\left(\tau-\tau^{\prime}\right)$ are single-electron Green functions for the subspaces characterized by the "statistical weights" $w_{p}=\mathrm{e}^{-\beta \Omega_{(p)}} / \sum_{q} \mathrm{e}^{-\beta \Omega_{(q)}}$ and our single-site atomic problem exactly splits into four subspaces $p=0,2, \downarrow, \uparrow$.

The fermionic Green's function in subspaces can be written as

$$
G_{\sigma(p)}\left(\omega_{n}\right)=\frac{1}{\Xi_{\sigma(p)}^{-1}\left(\omega_{n}\right)-J_{\sigma}\left(\omega_{n}\right)}, \quad \Xi_{\sigma(p)}^{-1}\left(\omega_{n}\right)=\mathrm{i} \omega_{n}+\mu_{\sigma}-U n_{\bar{\sigma}(p)}^{(0)}-\Sigma_{\sigma(p)}\left(\omega_{n}\right),
$$

where $n_{\sigma(p)}^{(0)}=-\mathrm{d} \lambda_{p} / \mathrm{d} \mu_{\sigma}=0$ for $p=0, \bar{\sigma}$ and 1 for $p=2, \sigma$ is an occupation of the state $|p\rangle$ by the electron with spin $\sigma$, and the self-energy $\Sigma_{\sigma(p)}\left(\omega_{n}\right)$ depends on the hopping integral $J_{\sigma^{\prime}}\left(\omega_{n^{\prime}}\right)$ only through quantities

$$
\Psi_{\sigma^{\prime}(p)}\left(\omega_{n^{\prime}}\right)=G_{\sigma^{\prime}(p)}\left(\omega_{n^{\prime}}\right)-\Xi_{\sigma^{\prime}(p)}\left(\omega_{n^{\prime}}\right) .
$$

Now, one can reconstruct the expressions for the grand canonical potentials $\Omega_{(p)}$ in the subspaces from the known structure of Green's functions:

$$
\Omega_{(p)}=\lambda_{p}-\frac{1}{\beta} \sum_{n \sigma} \ln \left(1-J_{\sigma}\left(\omega_{n}\right) \Xi_{\sigma(p)}\left(\omega_{n}\right)\right)-\frac{1}{\beta} \sum_{n \sigma} \Sigma_{\sigma(p)}\left(\omega_{n}\right) \Psi_{\sigma(p)}\left(\omega_{n}\right)+\Phi_{(p)},
$$

where $\Phi_{(p)}$ is some functional, such that its functional derivative with respect to $\Psi$ produces the self-energy: $\delta \Phi_{(p)} / \delta \Psi_{\sigma(p)}\left(\omega_{n}\right)=\Sigma_{\sigma(p)}\left(\omega_{n}\right)$. So, if we can find or construct the self-energy $\Sigma_{\sigma(p)}\left(\omega_{n}\right)$, we can find Green's functions and grand canonical potentials for the subspaces and, according to equations (4.5) and (4.6), we can solve atomic problems.

From the grand canonical potential (4.5) and (4.9) we get the following mean values

$$
n_{\sigma}=\sum_{p} w_{p} n_{\sigma(p)}, \quad n_{\sigma(p)}=n_{\sigma(p)}^{(0)}+\frac{1}{\beta} \sum_{n} \Psi_{\sigma(p)}\left(\omega_{n}\right)-\frac{\partial \Phi_{(p)}}{\partial \mu_{\sigma}},
$$

where in the last term the partial derivative is taken over $\mu_{\sigma}$ not in the chains (4.8).

For the Falicov-Kimball model $J_{\downarrow}\left(\omega_{n}\right)=0$ and, as a result, $\Sigma_{\uparrow(p)}\left(\omega_{n}\right) \equiv 0$ and $\Xi_{\uparrow(p)}\left(\omega_{n}\right)=g_{\uparrow(p)}\left(\omega_{n}\right)$ which immediately gives results of [4] (see also [13]).

For the Hubbard model, there is no exact expression for the self-energy but the set of equations (4.7) and (4.9) permits one to construct different self-consistent approximations. 


\section{Hartree-Fock approximation}

One of the possible approximations is to construct the equation for the self-energy in the following form:

$$
\Sigma_{\sigma(p)}\left(\omega_{n}\right)=\frac{1}{\beta} \sum_{n^{\prime}} U \Psi_{\bar{\sigma}(p)}\left(\omega_{n^{\prime}}\right)
$$

which, together with the expression for mean values

$$
n_{\sigma(p)}=n_{\sigma(p)}^{(0)}+\frac{1}{\beta} \sum_{n} \Psi_{\sigma(p)}\left(\omega_{n}\right),
$$

gives, for the Green's functions in the subspaces, an expression in the Hartree-Fock approximation:

$$
G_{\sigma(p)}\left(\omega_{n}\right)=\frac{1}{\mathrm{i} \omega_{n}+\mu_{\sigma}-U n_{\bar{\sigma}(p)}-J_{\sigma}\left(\omega_{n}\right)} .
$$

Now, the grand canonical potentials in the subspaces are equal

$$
\Omega_{(p)}=\lambda_{p}-\frac{1}{\beta} \sum_{n \sigma} \ln \left[1-J_{\sigma}\left(\omega_{n}\right) \Xi_{\sigma(p)}\left(\omega_{n}\right)\right]-U\left(n_{\sigma(p)}-n_{\sigma(p)}^{(0)}\right)\left(n_{\bar{\sigma}(p)}-n_{\bar{\sigma}(p)}^{(0)}\right)
$$

and the Green's function for the atomic problem (4.6) yield a four-pole structure, that, in contrast to the alloy-analogy solution $\Sigma_{\sigma(p)}\left(\omega_{n}\right)=0$, possesses the correct Hartree-Fock limit for a small Coulombic interaction $U \ll t$. On the other hand, in the same way as an alloy-analogy solution, it describes the metal-insulator transition with the change of $U$.

In [10] it was shown that the main contributions into the total spectral weight function $\rho_{\sigma}(\omega)=\frac{1}{\pi} \Im G_{\sigma}^{(a)}\left(\omega-\mathrm{i} 0^{+}\right)$come from the subspaces $p=0$ for the low electron concentrations $\left(n<\frac{2}{3}, \mu<0\right), p=2$ for the low hole concentrations $\left(2-n<\frac{2}{3}\right.$, $\mu>U)$ and $p=\sigma, \bar{\sigma}$ for the intermediate values. For the small electron or hole concentrations, the Green's function for the atomic problem (4.6) possess the correct Hartree-Fock limits as well. It was supposed that the Hubbard model describes strongly-correlated electronic systems that contain four components (subspaces). Subspaces $p=0$ and $p=2$ describe the Fermi-liquid component (electron and hole, respectively) which is dominant for the small electron and hole concentrations, when the chemical potential is close to the bottom of the lower band or top of the upper one ("overdoped regime" of high- $T_{\mathrm{c}}$ 's). On the other hand, subspaces $p=\uparrow$ and $\downarrow$ describe the non-Fermi-liquid (strongly correlated, e.g., RVB) component, which is dominant close to half-filling ("underdoped regime").

Here we consider possible magnetic orderings. At low temperatures, the $p=$ 0 and $p=2$ components for the low electron or hole concentrations are in the ferromagnetic state, while the non-Fermi-liquid one is antiferromagnetic (AF) close to half-filling, see figure 1. For the intermediate concentration values, the picture is very complicated, even frustrated. It is due to the fact that equations for the mean values (5.2) have several solutions in this region, which, on the other hand, are mutually connected with the dynamical mean field $J_{\sigma}\left(\omega_{n}\right)$. It is difficult to 

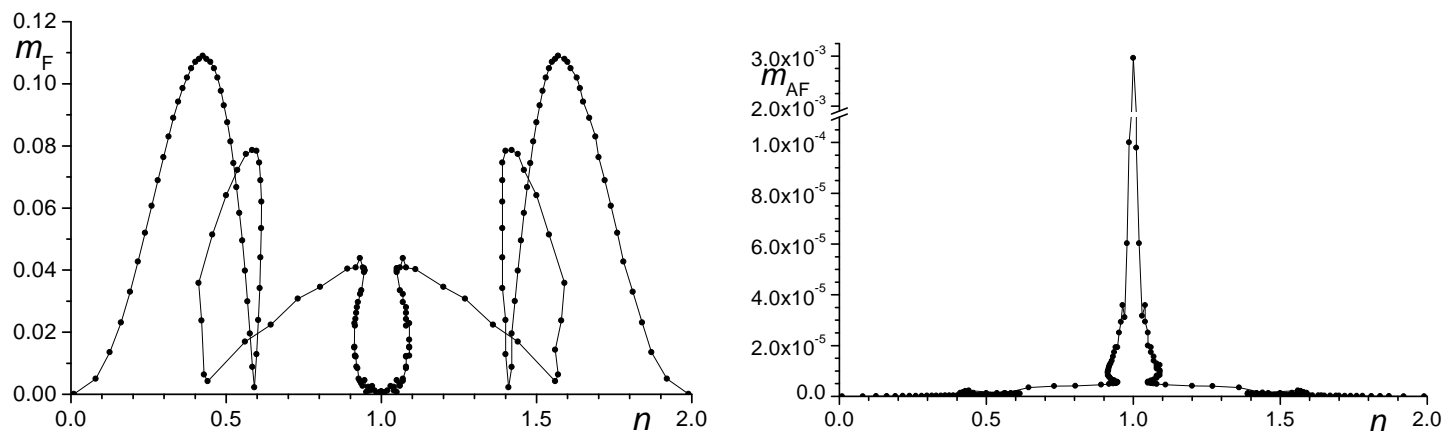

Figure 1. Ferromagnetic $m_{\mathrm{F}}$ and antiferromagnetic $m_{\mathrm{AF}}$ order parameters vs electron concentration for $U=1.56, T=0.14$.

determine the ground state for this, possibly "pseudo-gap", region, which is located between the ferromagnetic and antiferromagnetic phases.

In figure 2 we presented the phase diagram $(T, U)$ - the temperature of the AF ordering vs correlation energy $U$, which is in a qualitative agreement with the results of $[14,15]$ and reproduces the results of the Hartree-Fock theory and mean field approximation for $U \ll t$ and $U \gg t$, respectively. Our results for the AF critical temperature for small $U$ are higher than the one of the Quantum Monte Carlo simulations [14] by about a factor at three that describes the reduction of the Hartree-Fock solution by the lowest order quantum fluctuations [16].

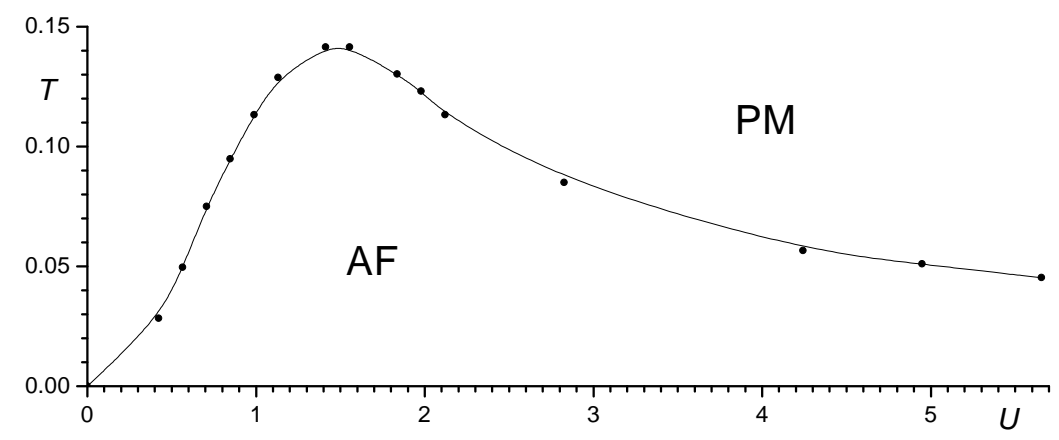

Figure 2. Phase diagram $(T, U)$ at half-filling $n=1$ (AF - antiferromagnetic phase, PM - paramagnetic phase).

\section{References}

1. Metzner W., Vollhardt D. // Phys. Rev. Lett., 1989, vol. 62, p. 324.

2. Izyumov Yu.A. // Uspekhi Fiz. Nauk, 1995, vol. 165, p. 403 [Physics-Uspekhi, 1995, vol. 38, p. 385].

3. Georges A., Kotliar G., Krauth W., Rosenberg M.J. // Rev. Mod. Phys., 1996, vol. 68, p. 13.

4. Brandt U., Mielsch C. // Z. Phys. B, 1989, vol. 75, p. 365; 1990, vol. 79, p. 295; 1991, vol. 82 , p. 37. 
5. Gebhard F. The Mott Metal-Insulator Transition: Models and Methods. Berlin, Springer-Verlag, 1997.

6. Cojocaru S.P., Moskalenko V.A. // Teor. Mat. Fiz., 1993, vol. 97, p. 270 [Theor. Math. Phys. USSR, 1993, vol. 97, p. 1290]; Moskalenko V.A., Kon L.Z. // Condens. Matter Phys., 1998, vol. 1(13), p. 23; Izyumov Yu.A., Chashchin N.I. // ibid., p. 41.

7. Slobodjan P.M., Stasyuk I.V. // Teor. Mat. Fiz., 1974, vol. 19, p. 423 [Theor. Math. Phys. USSR, 1974, vol. 19, p. 616].

8. Izyumov Yu.A., Skryabin Yu.N. Statistical Mechanics of Magnetically Ordered Systems. New York, Consultants Bureau, 1989.

9. Izyumov Yu.A., Letfulov B.M. // J. Phys.: Condens. Matter, 1990, vol. 2, p. 8905; Izyumov Yu.A., Letfulov B.M., Shipitsyn E.V., Bartkowiak M., Chao K.A. // Phys. Rev. B, 1992, vol. 46, p. 15697.

10. Shvaika A.M. // Phys. Rev. B, 2000, vol. 62, p. 2358.

11. Baym G., Kadanoff L.P. // Phys. Rev., 1961, vol. 124, p. 287; Baym G. // ibid., 1962, vol. 127, p. 1391.

12. Metzner W. // Phys. Rev. B, 1991, vol. 43, p. 8549.

13. Stasyuk I.V., Shvaika A.M. // J. Phys. Stud., 1999, vol. 3, p. 177.

14. Pruschke T., Cox D.L., Jarrell M. // Phys. Rev. B, 1993, vol. 47, p. 3553.

15. Kakehashi Y., Hasegawa H. // Phys. Rev. B, 1988, vol. 37, p. 7777; Rozenberg M.J., Kotliar G., Zhang X.Y. // ibid., 1994, vol. 49, p. 10181.

16. van Dongen P.G.J. // Phys. Rev. Lett., 1991, vol. 67, p. 757.

\title{
Наближення сильного зв'язку типу Хартрі-Фока в теорії динамічного середнього поля
}

\author{
А.М.Швайка \\ Інститут фізики конденсованих систем НАН України, \\ 79011 Львів, вул. Свєнціцького, 1 \\ Отримано 23 серпня 2000 р.
}

Для моделі Хаббарда побудовано термодинамічно самоузгоджену теорію, яка застосовна для довільних значень кулонівської кореляції $(U<\infty)$ в границі безмежної розмірності простору, коли повна допоміжна задача точно розпадається на чотири підпростори з різними "вакуумними станами". Наведено ряд аналітичних результатів для наближення Хартрі-Фока, коли отримується чотириполюсна структура для функцій Гріна: два полюси описують внески Фермі-рідинної компоненти, яка є феромагнітною і домінує при малих концентраціях електронів або дірок (“перелегований випадок” ВТНП), а інші два - внески від не-Фермі рідини, яка є антиферомагнітною і домінує поблизу половинного заповнення (“недолегований випадок”).

Ключові слова: теорія динамічного середнього поля, наближення Хартрі-Фока, модель Хаббарда, (анти)феромагнетизм, сильний зв'язок

PACS: $71.10 . F d, 71.15 . M b, 05.30 . F k, 71.27 .+a$ 